\title{
Remarks on the Iapetus' bulge and ridge
}

\author{
Leszek Czechowski and Jacek Leliwa-Kopystynski \\ Institute of Geophysics, Department of Physics, University of Warsaw, Ul. Pasteura 7, 02-093 Warszawa, Poland
}

(Received April 4, 2008; Revised May 26, 2012; Accepted December 18, 2012; Online published September 17, 2013)

\begin{abstract}
Iapetus is a medium sized icy satellite of Saturn. It has two spectacular features: the equatorial ridge (ER) and the abnormally large flattening. The flattening is usually explained in terms of large non-hydrostatic fossil equatorial bulge (EB) supported by a thick lithosphere. Here we show, building on the principle of isostasy, that EB and ER could be a result of low density roots underlying the lithosphere below the equator. The low density matter formed the layer over the core of the satellite. Such situation was unstable. The instability led to origin of axially symmetric plumes that formed equatorial bulge and equatorial ridge. So, we explain both: EB and ER in the frame of one hypothesis.
\end{abstract}

Key words: Iapetus; satellite, shape; satellites, surface; tectonics.

\section{Introduction}

Iapetus is a medium-sized icy satellite (MIS) of Saturn with two spectacular features: the geologically old equatorial ridge (ER) rising above the equatorial bulge (EB)Fig. 1. The topographic map of elevation of Iapetus confirms that ER is rising above EB-see e.g. figure 2 in Giese et al. (2008). The origin of both features still remains an enigma, nevertheless a few different hypotheses were presented. Some of them are supported by numerical models.

The interiors of MIS behave like viscous liquid in geological time scale, therefore the gravity and the centrifugal force give them a hydrostatic shape, i.e. an oblate ellipsoid (approximately a sphere with an equatorial bulgeEB) with flattening depending on period of rotation $T_{r}$ (flattening decreases if $T_{r}$ increases). Iapetus' flattening is equal to 0.046 and it corresponds $T_{r}=16$ hours although the present $T_{r}$ is 79.33 days. The initial $T_{r}$ was probably a few hours but tidal forces slowed the rotation down establishing synchronous rotation, i.e. $T_{r}=T_{\text {orbital }}$. The time of despinning $t_{\text {desp }}$ is proportional to $a^{-6}$ ( $a$ is the distance from Saturn) and depends also on the dissipation factor $Q . Q$ is high for rigid and elastic bodies and low for viscous, e.g. partially melted ones. Assuming typical $Q=100$ one obtains $t_{\text {desp }} \approx 240 \mathrm{Gyr}$, which is longer than the age of Iapetus! This shows that $Q$ was several orders of magnitude lower for a long time. However, the large flattening indicates the opposite, i.e. a thick and rigid lithosphere that could support the non-hydrostatic shape for billions of years.

These two facts are difficult to reconcile, although (Castillo-Rogez et al., 2007, 2009) found that they could be explained if the accretion of Iapetus had been completed shortly (3.5-4 Myr) after the formation of CAI (i.e. Calcium-Aluminium Inclusions in chondrites). More ad-

Copyright (C) The Society of Geomagnetism and Earth, Planetary and Space Sciences (SGEPSS); The Seismological Society of Japan; The Volcanological Society of Japan; The Geodetic Society of Japan; The Japanese Society for Planetary Sciences; TERRAPUB.

doi:10.5047/eps.2012.12.008 vanced model of Robuchon et al. (2010) further reduces the range of possible time of accretion and indicates that special Burgess rheology is necessary.

Other explanations of the origin of ER are also proposed:

1. Exogenic origin. ER is the build up of in-falling material from a ring or disk of the orbiting material (Ip, 2006; Levison et al., 2011; Walsh et al., 2011; Dombard et al., 2012). Walsh et al. (2011) present mechanical simulations of the ridge growth from an in-falling ring of debris. Dombard et al. (2012) consider formation of ER from a subsatellite created in a giant impact. According to them, the impact's ejecta formed the subsatellite. The tidal interaction between the bodies of the system eventually leads to origin of the ridge.

2. Contraction of Iapetus. Sandwell and Schubert (2010) have proposed the model that does not require high initial spin rate or the short lived radioactive isotopes (SLRI). Initially Iapetus is a slightly oblate spheroid with the porosity higher than $10 \%$. Radioactive heating by long-lived isotopes warms the interior to about $200 \mathrm{~K}$, at which point the interior becomes ductile and compacts by $10 \%$, while the $120 \mathrm{~km}$-thick external shell remains solid. The shell deforms to match the reduced volume of the interior. The deformation occurs along the equator, probably as a result of a thinner shell below the equator. To maintain this non-equilibrium shape, the thickness of the shell must exceed $120 \mathrm{~km}$.

3. Thermal convection inside Iapetus driven by a postaccretion heating and short-living radioactive isotopes (Czechowski and Leliwa-Kopystynski, 2005, 2008).

In the present paper we follow some suggestions of Czechowski and Leliwa-Kopystyński (2008), i.e. we try to explain the Iapetus shape and existence of ER without assuming a large non-hydrostatic equatorial bulge. Instead of purely thermal convection we propose gravitational differentiation followed by convection driven by difference of the density. 


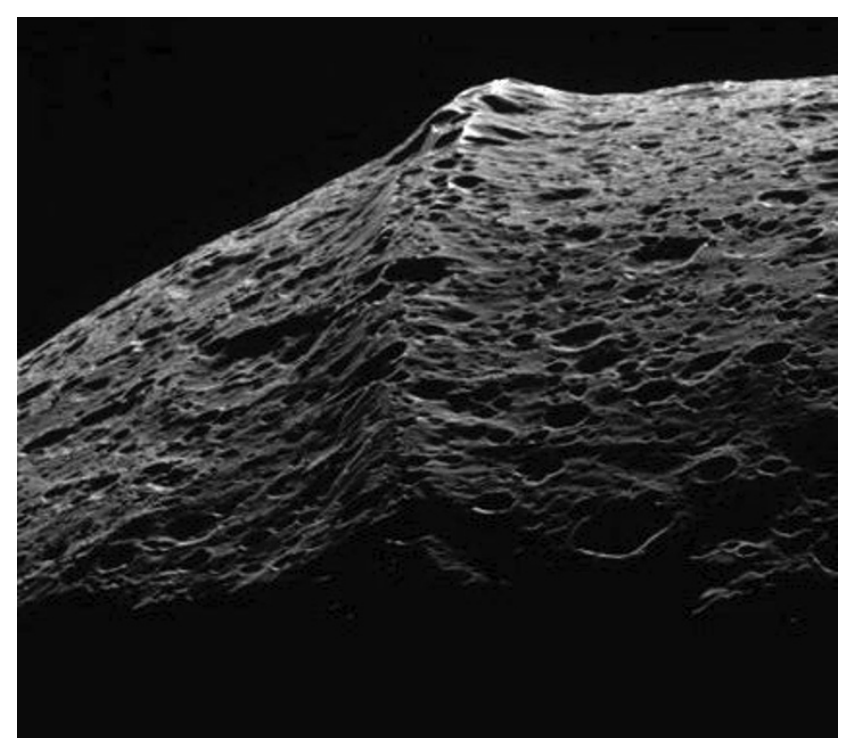

Fig. 1. The equatorial ridge on Iapetus seen from Cassini probe. Distance $62,331 \mathrm{~km}$. NASA.

\section{Isostasy and Scale of Differentiation}

Let us note that the considered here Cassini data concern the geometric shape only (e.g. Giese et al., 2008; Thomas et al., 2007; Thomas, 2010). The comparison of this shape with the shape of equipotential surface, i.e. the geoid, is necessary to prove that the flattening is non-hydrostatic. Most of terrestrial mountains are in isostatic equilibrium, i.e. they are underlain by 'roots' formed from low density matter. The gravity acting on the elevated parts is compensated by buoyancy force acting on the roots. The isostatic equilibrium is a result of the fluid-like properties of the rocks in the Earth's mantle.

The rheology of icy satellites is similar to terrestrial rocks although for different range of temperature and pressure. The uppermost layer ('lithosphere') is elastic for small deformation and brittle for large deformations. It means that mountains on the surface could exist for billions of year. The medium below the lithosphere is also solid but for very slow geologic processes it behaves like a viscous fluid.

The volume percentage of silicate component in Iapetus is substantially below $60 \%$. It means that rheological properties of its interior are determined by the icy component (Roscoe, 1952; Schubert et al., 1986). Theoretical and experimental results indicate the following formulae for the viscosity of the satellite interior:

$$
\eta(T)=\eta_{x} \sigma^{(1-i)} \exp \left(\frac{E}{R T}\right),
$$

where $\eta_{x}$ is a constant, $\sigma$ is the second invariant of the deviatoric stress tensor, $i$ is the power law index $(i=1$ corresponds to Newtonian fluid), $E$ is the activation energy of the dominant mechanism of deformation, and $R=8.314$ $\left[\mathrm{J} \mathrm{K}^{-1} \mathrm{~mole}^{-1}\right.$ ] is the universal gas constant (Forni et al., 1991; Goldsby and Kohlstedt, 1997; Durham et al., 1998; McKinnon, 1998). Parameters $\eta_{x}, E$ and $i$ depend on many factors; e.g. size of ice crystal, content of gases, size of mineral grains etc (see also: Christensen, 1984; Dumoulin et al., 1999). The exact values of these parameters are not of special importance for our hypothesis. The main conclusion is that the interior of Iapetus behaves (or behaved during origin of ER) like a fluid. It means that the isostasy is (or was) valid also for Iapetus and EB is some sort of $700 \mathrm{~km}$ wide rise underlain by low-density roots-Fig. 2.

The low density roots are a result of gravitational differentiation of the satellite's matter. Initially, the matter was a mixture of silicates, ices (of $\mathrm{H}_{2} \mathrm{O}$ and other volatiles) and organic matter (e.g. Cruikshank et al., 2008) of average density $\rho_{\mathrm{av}}=1083 \mathrm{~kg} \mathrm{~m}^{-3}$. The melting leads to separation of denser silicates (we assume here that $\rho_{\text {sil }}=3510 \mathrm{~kg} \mathrm{~m}^{-3}$ )
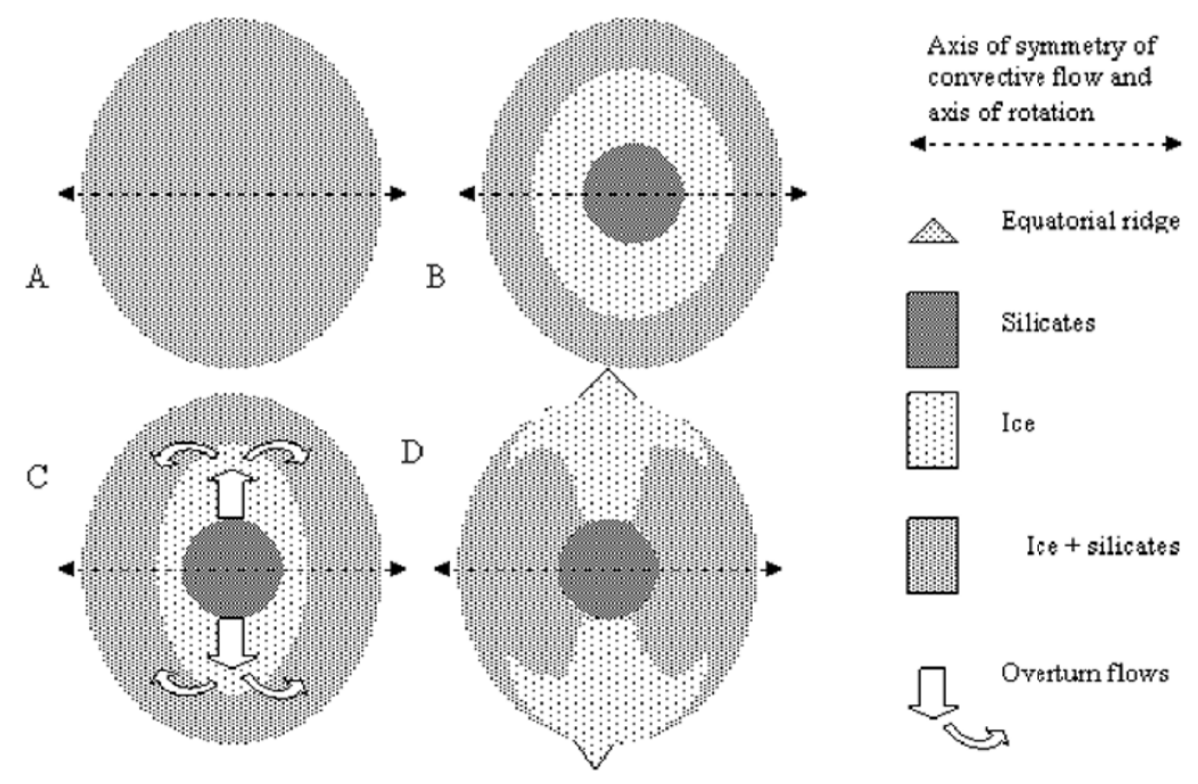

Fig. 2. Evolution of Iapetus according to our hypothesis: A-homogeneous satellite after accretion. B-high density core enclosed by the ice layer as a result of melting, gravitational differentiation and freezing. The ice thickness is latitude dependent as a result of inertial forces. $\mathrm{C}-$ gravitational overturn by axially symmetric flow. D-present structure of Iapetus. 
$\mathbf{A}$
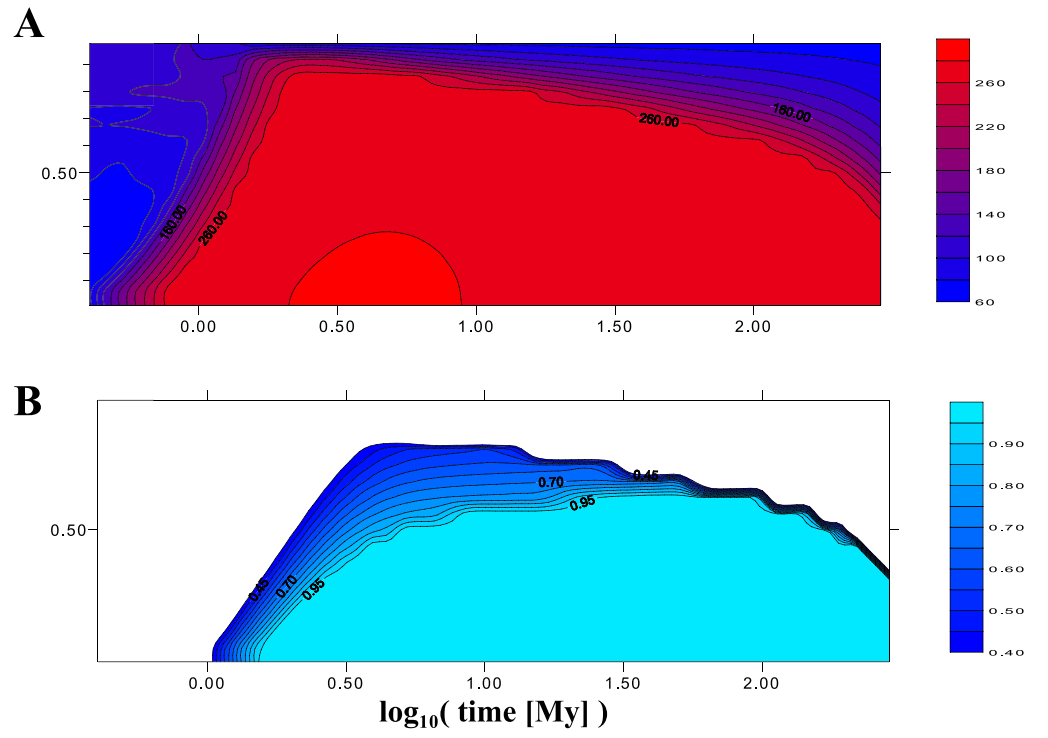

$\mathbf{C}$

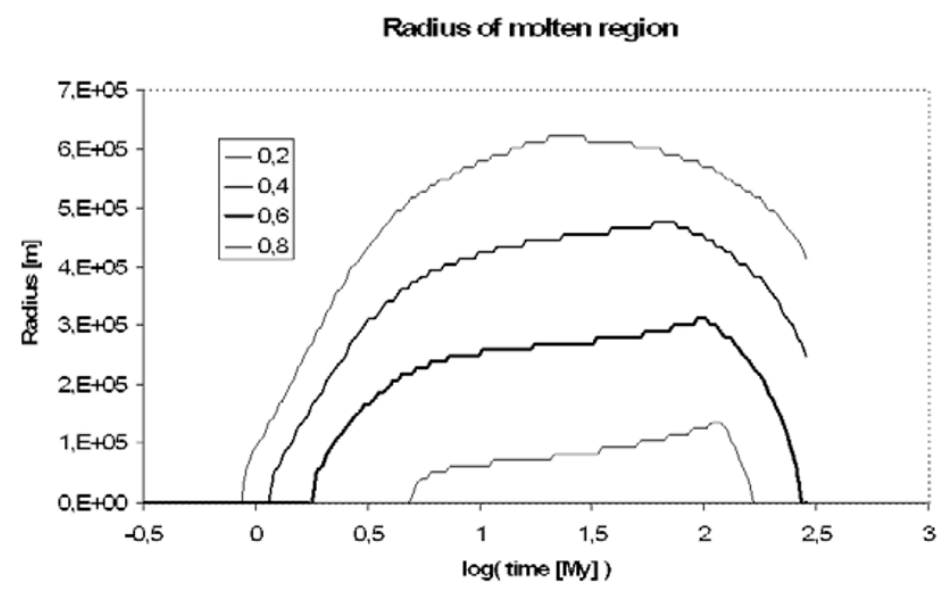

Fig. 3. Panels A and B: An example of thermal and melting history of Iapetus according to our model for $t_{\text {ini }}=0.4$ My, $t_{\mathrm{acr}}=1 \mathrm{My}, \eta_{0}=10^{12}$ Pa s. The vertical axis gives $r / R_{\text {sat }}$, the horizontal axis gives $\log _{10} t$, where $t$ [My] is the time from the beginning of accretion. Panel A presents isotherms in Kelvin. Panel B presents fraction of latent heat supplied to the matter (1 means that matter is molten). Note substantial phase shift between temperature distribution and melting. Panel C: Radius of the molten region versus time for 4 different $t_{\text {ini }}\left(\right.$ see legend), $t_{\mathrm{ac}}=1 \mathrm{My}$ and $\eta_{0}=10^{12} \mathrm{~Pa}$ s. Note that partial melting of the satellite is possible for limited range of parameters only. For $t_{\text {ini }}>1 \mathrm{My}$ there is not melting at all, while for $t_{\text {ini }}<0.2$ My the whole satellite is molten.

from ices $\rho_{\text {ice }}=940 \mathrm{~kg} \mathrm{~m}^{-3}$. If EB and the roots are built mainly of the ices, then the isostasy requires:

$$
h \rho_{\text {ice }}=\Delta \rho w,
$$

where $h$ is the height above the reference sphere, $w$ is depth of the roots and $\Delta \rho=\rho_{\mathrm{av}}-\rho_{\text {ice }}$ is $143 \mathrm{~kg} \mathrm{~m}^{-3}$. So, $w / h=\rho_{\text {ice }} / \Delta \rho \approx 7$, i.e. the roots are 7 times thicker than the height of EB. At the equator $h=12 \mathrm{~km}$, so there $w=7 \times 12=84 \mathrm{~km}$. Of course, $w$ decreases with increasing latitude. Under ER (that is elevated about 18 $\mathrm{km}$ above EB) the roots are as deep as $210 \mathrm{~km}$. The total volume of $\mathrm{EB}$ and the roots must be at least $24 \%$ of Iapetus volume $(\mathrm{EB}-3 \%$, and the roots-21\%). So, at least $24 \%$ of Iapetus must have been a subject to differentiation.

\section{Model of Thermal History and Differentiation}

Differentiation starts when the temperature exceeds the melting temperature of the ice. Calculations (e.g. CastilloRogez et al., 2007) indicate that temperature in the substantial part of Iapetus (30-50\%) was high enough for melting. However their calculations do not include the role of convection. Czechowski (2012) develops the model based on the finite difference method combined with parameterized model of convection. It includes solid state convection, melting, convection in molten region and differentiation. The heating from the decay of the short-lived and the long-lived radioactive isotopes are included as well as the heat of accretion. The model has 3 basic parameters: (i) $t_{\text {ini }}$ time from formation of CAI to beginning of accretion, (ii) $t_{\text {acr }}$ duration of accretion, (iii) $\eta_{0}$ viscosity of the satellite interior at the temperature of melting (but before melting).

The full description of the model is given in Czechowski 
$\mathbf{A}$

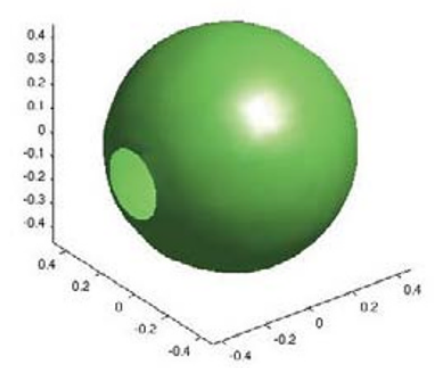

C

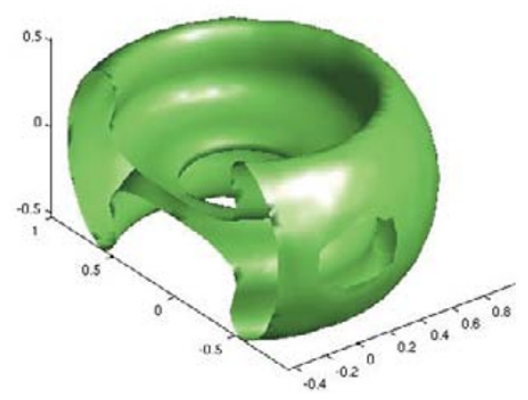

B

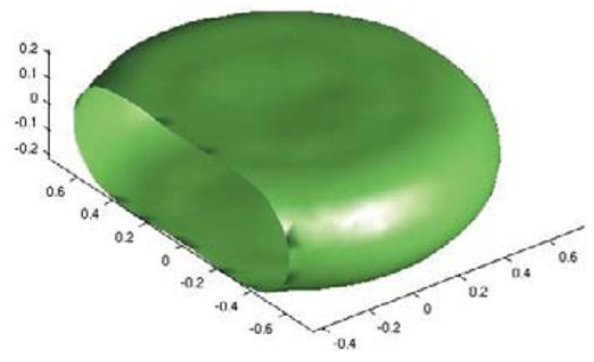

D

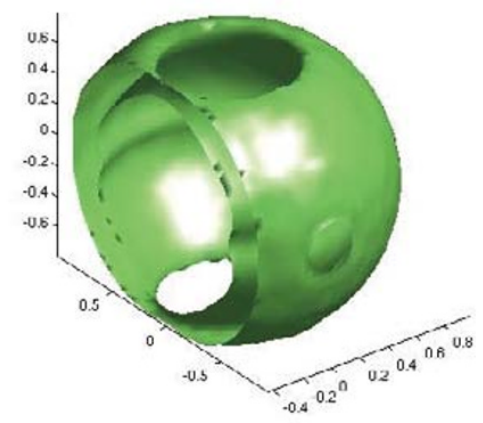

Fig. 4. Model of the gravitational overturn according to the presented hypothesis for $R a=200000$. A-Initial distribution of light fraction in a spherical layer. B-light matter forms an axially symmetric plume. C and D-the plume reaches the surface and forms equatorial bulge and equatorial ridge.

(2012). We present a few results of this model calculated for Iapetus. Figure 3(A) and (B) give thermal and melting history of the first $300 \mathrm{My}$ of Iapetus for the following values of the parameters: $t_{\text {ini }}=0.4 \mathrm{My}, t_{\mathrm{acr}}=1 \mathrm{My}, \eta_{0}=10^{12}$ $\mathrm{Pa}$ s. Panel A presents isotherms in Kelvin, panel B presents region where the melting temperature is reached and melting is started. Isolines give fraction of the latent heat of melting supplied to the matter (i.e. 1 means full melting). Note substantial difference between temperature distribution and melting distribution. Panel $\mathrm{C}$ presents radius of the molten region versus time for $t_{\mathrm{acr}}=1 \mathrm{My}, \eta_{0}=10^{12} \mathrm{~Pa} \mathrm{~s}$, and for a few different values of $t_{\text {ini }}=0.2,0.4,0.6,0.8 \mathrm{My}$.

Our considerations above indicate that at least $24 \%$ of Iapetus must be melted. It corresponds to the radius: $r_{\text {mel }}=$ $(0.24))^{1 / 3} R_{\text {sat }}=0.62 R_{\text {sat }}$. Figure $3(\mathrm{C})$ indicates that such $r_{\text {mel }}$ is possible for $t_{\text {ini }}$ in the range $0.4-0.6 \mathrm{My}$.

\section{Molten Region and the Coriolis Force}

The melting leads to gravitational differentiation. After differentiation, the silicates form the central core, while the liquid forms a molten layer around the core. The thermal convection operates in the molten region. This convection is not a solid state convection where Prandtl number $\mathrm{Pr}=$ $\eta / \kappa=O\left(10^{20}\right)$ but convection in liquid with $\mathrm{Pr}=O(10)$. Such convection is sensitive to the Coriolis force:

$$
\boldsymbol{F}_{\text {Cor }}=2 \rho \boldsymbol{v} \times \boldsymbol{\omega},
$$

where $\omega$ is the angular velocity of the satellite rotation, and $v$ is the velocity vector of the fluid. The direction and magnitude of $\boldsymbol{F}_{\text {Cor }}$ depend on the vectors $\boldsymbol{v}$ and $\boldsymbol{\omega}$ and consequently on the latitude. This leads to the latitude dependent pattern of convective flow and to latitude dependent heat transport (cooling). The ratio of Coriolis force to viscous force is of the order of (Czechowski, 2012):

$$
\frac{\left|F_{\mathrm{Cor}}\right|}{\left|F_{\mathrm{v}}\right|}=\frac{2 \rho \omega s^{2}}{\eta},
$$

where $s$ is the spatial scale of the velocity changes. Of course, $s$ must be smaller than the size of molten region. Substituting $\omega=9.17 \times 10^{-7} \mathrm{rad} \mathrm{s}^{-1}$ (present angular velocity of Iapetus), $\rho=1000 \mathrm{~kg} \mathrm{~m}^{-3}, \eta=10^{-3} \mathrm{~Pa} \mathrm{~s}, s=$ $10^{4} \mathrm{~m}$, we get $O\left(10^{8}\right)$. The Coriolis force is much larger than the viscous force in the molten region. Consequently, one can expect latitude dependent flow.

\section{The Model of the Overturn}

After differentiation and freezing of the molten region, the silicates form the core while ices form a layer around the core-Fig. 2(B). It is an unstable situation, because low density ice $\left(\rho_{\text {ice }}\right)$ underlies the matter of higher density $\left(\rho_{\mathrm{av}}\right)$. It results in the overturn (e.g. Grasset and Sotin, 1996). The proper system of equations describing the process is first introduced in geodynamics by Czechowski (1984). More details of equations are given in Czechowski (1993). The buoyancy resulting from the difference of density $\Delta \rho$ is the main driving force. The dimensionless equations of motion and transport are:

$$
\begin{aligned}
& 0=-\nabla p+\nabla \cdot\left[\eta\left(\nabla v+(\nabla v)^{T}\right]+R a i_{r},\right. \\
& \frac{\partial Z}{\partial t}+\boldsymbol{v} \cdot \nabla Z=\nabla^{2} Z
\end{aligned}
$$

where $p$ is the pressure, $v$ is the velocity field, $Z$ is the dimensionless fraction of the low density matter $(0<Z<$ 
1). The $R a$ denotes a dimensionless group analogous to the Rayleigh number in thermal convection. $R a$ is defined as follow:

$$
R a=\frac{g \Delta \rho R_{\mathrm{Sat}}^{3}}{\eta \kappa},
$$

where $\eta$ is the viscosity [Pa s] and $\kappa\left[\mathrm{m}^{2} \mathrm{~s}^{-1}\right]$ is the thermal diffusivity of the satellite's interior. L. Czechowski developed the 3D numerical model of the overturn and performed calculations described below. Four panels in Fig. 4 present consecutive phases of the motion for $R a=200000$ and for free of stress upper and lower boundary conditions. Initially, the low density matter is concentrated in a spherical layer. This layer is slightly thicker below the equatorial region according to formula $0.00001 R_{\text {Sat }} \sin (l)$ where $l$ is the latitude. It simulates the role of latitude dependent Coriolis force. Although the disturbance is minimal the resulting motion is almost perfectly axially symmetric. The spherical layer forms a symmetric plume (B). The plume reaches the surface and forms the equatorial bulge (C and $D)$. In some places the plume could deform and penetrate the lithosphere forming the equatorial ridge.

After the overturn the present stable state was established. Let us note that axial symmetry of the flow is a consequence of latitude dependent thickness of the ice layer. In the absence of this factor the uprising matter would form several small separate plumes. It is analogous to thermal convection when initial pattern consists of small downward currents (e.g. Robuchon et al., 2010).

The thermal convection evolves to the steady state (e.g. Czechowski and Leliwa-Kopystynski, 2005). For moderate values of the Rayleigh number the steady state often forms two-cell pattern consisting of two torus-like cells with a common region of upward motion (below the equator) and two regions of downward motion (below the polar regions). The process of displacement of the ice layer is too short for similar evolution. When the whole ice concentrates close to the surface, the stability is restored and the motion stops. Then the isostatic vertical motion forms the EB (density of the upper layer around the equator is lower than the density in the polar regions).

The substantial redistribution of mass inside the satellite changes its moments of inertia and consequently its rotation axis. However in the case of the considered overturn the redistribution was axially symmetric and the rotation axis kept its direction.

There are a few advantages of our hypothesis. It explains both: EB and ER in the frame of one hypothesis. The lithosphere is not treated as an ideal layer that can support large (and large scale) non-hydrostatic load for billions of years. The lithosphere could be a realistic one (e.g. fractured by large impacts during HBE). Note, that strength of the intack rock is not a crucial parameter in the basic theory of thrust faulting (e.g. Watts, 2001, pp. 253-271; Turcotte and Schubert, 2002, pp. 343-350). Usually the medium contains many pre-existing faults. Under the tectonic stresses those pre-existing zones of weakness are activated. The effective strength of the rock layer depends mostly on the coefficient of friction and on weight of the rock layer. Let us note also, that our hypothesis does not require any special reological properties of the interior of Iapetus (compare e.g. Robuchon et al., 2010).

All hypotheses of the origin of ER require the material building the equatorial ridge is of different composition than the average of the satellite. Endogenic (e.g. volcanic) origins assume differentiation of the primitive matter. It means that material of ER is enriched in volatiles. The hypotheses assuming origin of ER from debris put into orbit require enrichment of $\mathrm{ER}$ in non-volatiles as a result of heating by impacts. So, the bulk composition of ER could indicate way of origin. Unfortunately most of the surface of Iapetus is covered by material that probably is different than material of underlying layer. In our hypothesis most of the roots of EB could be covered by the crust of primitive composition (see Fig. 2).

Why the excess flattening and the equatorial ridge are found on the Iapetus only? Let us consider the situation of other MIS of Saturn. Iapetus is a specific satellite for many reasons (e.g. Mosqueira and Estrada, 2005; Giese et al., 2008). It is the most distant of all other MIS of Saturn. It accreted in other part of nebula, so it could include more volatiles. Moreover, the uniform model of all MIS of Saturn developed by Czechowski (2006, 2009) indicates problems of including Iapetus. Our hypothesis allows also for other explanations. Czechowski (2012) indicates that partial differentiation is possible only for small range of parameters of the model. For low $t_{\text {ini }}$ usually the whole celestial body is differentiated. For large $t_{\text {ini }}$ there are not melting and no differentiation at all. For both cases densities of the crust in the equatorial region and polar region would the same, so formation of EB and ER would be not possible. It is also possible that ER is not unique. Some structures on other satellites could be analogous to ER (Czechowski and Leliwa-Kopystynski, 2005).

We hope that Cassini mission will provide data about gravity of Iapetus necessary to discriminate between hypotheses of hydrostatic and non-hydrostatic flattening.

Acknowledgments. This work was partially supported by the Polish Ministry of Education and Science (grant 4036/B/H03/2010/39) and National Science Centre (grant 2011/01/B/ST10/06653). Computer resources of Interdisciplinary Centre for Mathematical and Computational Modeling (ICM) of Warsaw University are also used in the research.

\section{References}

Castillo-Rogez, J., D. L. Matson, C. Sotin, T. V. Johnson, J. I. Lunine, and P. C. Thomas, Iapetus' geophysics: Rotation rate, shape, and equatorial ridge, Icarus, 190, 179-203, 2007.

Castillo-Rogez, J., T. V. Johnson, M. H. Lee, N. J. Turner, D. L. Matson, and J. Lunine, ${ }^{26} \mathrm{Al}$ decay: Heat production and a revised age for Iapetus, Icarus, 204, 658-662, 2009.

Christensen, U., Convection with pressure and temperature-dependent non-Newtonian rheology, Geophys. J. R. Astron. Soc., 77, 343-84, 1984. Cruikshank, D. P. et al., Hydrocarbons on Saturn's satellites Iapetus and Phoebe, Icarus, 193, 334-343, 2008.

Czechowski, L., The numerical model of the Earth's mantle convection with gravitational differentiation, Acta Geophys. Pol., XXIX(3), 207$215,1984$.

Czechowski, L., Theoretical approach to mantle convection, in Dynamics of the Earth's Evolution, edited by R. Teisseyre, L. Czechowski, and J. Leliwa-Kopystyński, pp. 161-271, PWN-Elsevier Sc. Pub., Amsterdam, 1993.

Czechowski, L., Parameterized model of convection driven by tidal and 
radiogenic heating, Adv. Space Res., 38, 788-793, 2006.

Czechowski, L., Uniform parameterized theory of convection in medium sized icy satellites of Saturn, Acta Geophys., 57(2), 548-566, 2009.

Czechowski, L., Thermal history and differentiation of Rhea, Acta Geophys., 60(4), 1192-1212, 2012.

Czechowski, L. and J. Leliwa-Kopystyński, Convection driven by tidal and radiogenic heating in medium size icy satellites, Planet. Space Sci., 53, 749-769, 2005.

Czechowski, L. and J. Leliwa-Kopystyński, The Iapetus's ridge: Possible explanations of its origin, Adv. Space Res., 42, 61-69, 2008.

Dombard, A. J., F. Andrew, A. F. Cheng, W. B. McKinnon, and J. P. Kay, Delayed formation of the equatorial ridge on Iapetus from a subsatellite created in a giant impact, J. Geophys. Res., 117, E03002, doi:10.1029/2011 je004010, 2012.

Dumoulin, C., M.-P. Doin, and L. Fleitout, Heat transport in stagnant lid convection with temperature- and pressure-dependent Newtonian or non-Newtonian rheology, J. Geophys. Res., 104, 12,759-12,777, 1999.

Durham, W. B., S. H. Kirby, and L. A. Stern, Rheology of planetary ices, in Solar System Ices, edited by Schmitt, B., C. de Bergh, and M. Festou, pp. 63-78, Kluwer Academic Publishers, Dordrecht, The Netherland, 1998.

Forni, O., A. Coradini, and C. Federico, Convection and lithospheric strength in Dione, an icy satellite of Saturn, Icarus, 94, 232-245, 1991.

Giese, B., T. Denk, G. Neukum, T. Roatsch, P. Helfenstein, P. C. Thomas, E. P. Turtle, A. McEwen, and C. C. Porco, The topography of Iapetus' leading side, Icarus, 193, 359-371, 2008.

Goldsby, D. L. and D. L. Kohlstedt, Grain boundary sliding in fine-grained Ice-I, Scr. Mater., 37, 1399-1405, 1997.

Grasset, O. and C. Sotin, The cooling rate of a liquid shell in Titan's interior, Icarus, 123, 101-112, 1996.

Ip, W.-H., On a ring origin of the equatorial ridge of Iapetus, Geophys. Res. Lett., 33, L16203, doi:10.1029/2005GL025386, 2006.

Levison, H. F., K. J. Walsh, A. C. Barr, and L. Dones, Ridge formation and de-spinning of Iapetus via an impact-generated satellite, Icarus, 214, 773-778, 2011.
McKinnon, W. B., Geodynamics of icy satellites, in Solar System Ices, edited by Schmitt, B., C. de Bergh, and M. Festou, pp. 525-550, Kluwer Academic Publishers Dordrecht, The Netherlands, 1998.

Mosqueira, I. and P. R. Estrada, On the origin of the Saturnian satellite system: Did Iapetus form insitu?, Lunar and Planetary Science XXXVI, 1951.pdf, 2005.

Porco, C. C. and 34 colleagues, Cassini Imaging Science: Initial Results on Phoebe and Iapetus, Science, 307, 1237-1242, 2005.

Robuchon, G., G. Choblet, G. Tobie, O. Cadek, C. Sotin, and O. Grassem, Coupling of thermal evolution and despinning of early Iapetus, Icarus, 207, 959-971, 2010.

Roscoe, R., The viscosity of suspensions of rigid spheres, British J. Appl. Phys., 3, 267-269, 1952.

Sandwell, D. and G. Schubert, A contraction model for the flattening and equatorial ridge of Iapetus, Icarus, 210, 817-822, 2010.

Schubert, G., T. Spohn, and R. T. Reynolds, Thermal histories, compositions and internal structures of the moons of the solar system, in Satellites, edited by Burns, J. A. and M. S. Matthews, pp. 224-292, The University of Arizona Press, Tucson, 1986.

Thomas, P. C., Sizes, shapes, and derived properties of the Saturnian satellites after the Cassini nominal mission, Icarus, 208, 395-401, 2010.

Thomas, P. C. and 14 colleagues, Shapes of the Saturnian ice satellites and their significance, Icarus, 190, 573-584, 2007.

Turcotte, D. L. and G. Schubert, Geodynamics, 343-350, Cambridge Univ Press, Cambridge, U.K., 2002.

Watts, A. B., Isostasy and Flexure of the Lithosphere, pp. 253-271, Cambridge Univ. Press., Cambridge, U.K., 2001.

Walsh, K. J., H. F. Levison, D. C. Richardson, and S. R. Schwartz, Building the Ridge of Iapetus: Modeling an In-Falling Ring. EPSC Abstracts Vol. 6, EPSC-DPS2011-1527-1, EPSC-DPS Joint Meeting, 2011. (http://meetingorganizer.copernicus.org/EPSC-DPS2011/EPSCDPS2011-1527-1.pdf)

L. Czechowski (e-mail: lczecho@op.pl) and J. Leliwa-Kopystynski 